\title{
A NONVANISHING THEOREM \\ FOR DERIVATIVES OF AUTOMORPHIC $L$-FUNCTIONS WITH APPLICATIONS TO ELLIPTIC CURVES
}

\author{
DANIEL BUMP, SOLOMON FRIEDBERG AND JEFFREY HOFFSTEIN
}

1. A brief history of nonvanishing theorems. The nonvanishing of a Dirichlet series $\sum a(n) n^{-s}$, or the existence of a pole, at a particular value of $s$ often has applications to arithmetic. Euler gave the first example of this, showing that the infinitude of the primes follows from the pole of $\zeta(s)$ at $s=1$. A deep refinement was given by Dirichlet, whose theorem on primes in an arithmetic progression depends in a fundamental way upon the nonvanishing of Dirichlet $L$-functions at $s=1$.

Among the many examples of arithmetically significant nonvanishing results in this century, one of the most important is still mostly conjectural. Let $E$ be an elliptic curve defined over Q: the set of all solutions to an equation $y^{2}=x^{3}-a x-b$ where $a, b$ are rational numbers with $4 a^{3}-27 b^{2} \neq$ 0 . Mordell showed that $E(\mathbf{Q})$ may be given the structure of a finitely generated abelian group. The Birch-Swinnerton-Dyer Conjecture asserts that the rank of this group is equal to the order of vanishing of a certain Dirichlet series $L(s, E)$ as $s=1$ - the center of the critical strip-and that the leading Taylor coefficient of this $L$-function at $s=1$ is determined in an explicit way by the arithmetic of the elliptic curve. We refer to the excellent survey article of Goldfeld [5] for details.

In 1977, Coates and Wiles [3] proved the first result towards the BirchSwinnerton-Dyer conjecture. The conjecture implies that if the $L$-series of $E$ does not vanish at 1 , then the group of rational points is finite. Coates and Wiles proved this last claim in the special case that $E$ has complex multiplication (nontrivial endomorphisms). In this note, we announce a nonvanishing theorem which, together with work of Kolyvagin and Gross-Zagier, implies that $E(\mathbf{Q})$ is finite when $L(1, E) \neq 0$ for any modular elliptic curve $E$. (A modular elliptic curve is one which may be parametrized by automorphic functions. Deuring proved that all elliptic curves with complex multiplication are modular; Taniyama and Weil have conjectured that indeed all elliptic curves defined over $\mathbf{Q}$ are modular.)

Before giving details of our theorem, we mention several other nonvanishing theorems and arithmetic applications. The following discussion is necessarily not a complete survey

Shimura showed that there is a correspondence between modular forms $f$ of even weight $k$ and modular forms $\tilde{f}$ of half-integral weight $(k+1) / 2$.

Received by the editors December 13, 1988 and, in revised form, February 20, 1989.

1980 Mathematics Subject Classification (1985 Revision). Primary 11G40; Secondary $11 \mathrm{~F} 11,11 \mathrm{~F} 46,11 \mathrm{~F} 67$.

This work was supported by grants from the National Science Foundation.

(C) 1989 American Mathematical Society $0273-0979 / 89 \$ 1.00+\$ .25$ per page 
He also raised the question of precisely which forms $f$ are in the image of the correspondence. Waldspurger $[16,17]$ proved that $f$ is in the image if and only if the twist $L(s, f, \chi)$ of $f$ by some quadratic character $\chi$ is nonzero at $s=k / 2$. Moreover, he proved that the squarefree Fourier coefficients of $\tilde{f}$ are then essentially the values of $L(k / 2, f, \chi)$ as $\chi$ runs through the quadratic Dirichlet characters.

Waldspurger's result is not unconnected with the Birch-SwinnertonDyer conjecture, since if the Taniyama-Weil conjecture is true, an elliptic curve $E$ is associated with the modular form $f$ of weight two such that the $L$-function of $E$ is the same as the $L$-function of $f$. In this case, the nonvanishing of $L(1, f)$ is related both to the existence of an automorphic form of weight $3 / 2$ (by Waldspurger's theorem), and to the finiteness of $E(\mathbf{Q})$ (by the Birch-Swinnerton-Dyer conjecture). Tunnell [15] applied this connection to solve the classical congruent number problem, which asks which integers are the areas of rational right triangles, using Waldspurger's Theorem and the Coates-Wiles Theorem.

Waldspurger's characterization of the image of the Shimura correspondence is but one example of a more general phenomenon related to theta correspondences. If it is possible to embed two groups into the metaplectic group - the double cover of a symplectic group-in such a way that they centralize each other, there may be a theta kernel which induces a lifting of automorphic forms on the one group to the other. (Cf. Howe [9].) Then it is typical for the image of the correspondence to have a characterization in terms of properties of $L$-functions. As another example, there is a correspondence between automorphic forms on $\mathrm{Sp}(4)$ and on $G L(4)$. Jacquet, Piatetski-Shapiro and Shalika [10] proved that an automorphic form on $G L(4)$ is in the image of this correspondence if and only if the exterior square $L$-function-a certain Euler product of degree six-has a pole at $s=1$. Blasius, Clozel and Ramakrishnan [1] applied this result to prove that Maass forms of eigenvalue $1 / 4$ have algebraic Fourier coefficients.

Another source of motivation for nonvanishing theorems is that such results were needed by Shimura for his theory of algebraicity of periods of modular forms. With such motivation, Shimura [14] showed that given a weight $k$ modular form $f$, there is some character of finite order $\chi$ such that $L(1, f, \chi)$ is not zero. This was refined by Rohrlich [13], who proved that for $f$ an arbitrary automorphic form on $P G L(2)$ and for arbitrary $s$, there exists a character $\chi$ of finite order such that $L(s, f, \chi) \neq 0$.

As Waldspurger's results indicate, it is often desirable to have nonvanishing theorems for twists by quadratic characters. One technique for proving that an $L$-function has a nonvanishing quadratic twist exploits the occurrence of quadratic twists of the $L$-function in the Fourier coefficients of Eisenstein series on the metaplectic group. Perhaps the first work of this type is the paper of Goldfeld, Hoffstein and Patterson [7], to be discussed below. A later paper of Goldfeld and Hoffstein [6] obtained mean value estimates for quadratic Dirichlet $L$-functions by these methods. Hoffstein, in unpublished work, used the occurrence of cubic Dirichlet $L$-functions in the Whittaker models of Eisenstein series in the residual spectrum on the three-fold cover of $G L(3)$ to obtain similar mean value estimates. 
The present work, and our related paper [2], use a similar technique.

2. A nonvanishing theorem for derivatives of $L$-functions. Let $f$ be a cuspidal new-form of even weight $k$ and trivial character for $\Gamma_{0}(N)$. Let $\Lambda(s, f)=N^{s / 2}(2 \pi)^{-s} \Gamma(s) L(s, f)$ be the $L$-function of $s$ with gamma factor. We have a functional equation $\Lambda(s, f)=\varepsilon \Lambda(k-s, f)$, where $\varepsilon= \pm 1$.

THEOREM. Assume that the sign $\varepsilon$ in the functional equation of $f$ is +1 . Let $S$ be a finite set of primes including those dividing $N$. Then there exist infinitely many imaginary quadratic fields $K=\mathbf{Q}(\sqrt{D})$ of discriminant $D<$ 0 prime to $N$ such that the twisted $L$-function $L\left(s, f, \chi_{D}\right)$ has a first order zero at $s=k / 2$, where $\chi_{D}$ is the quadratic Dirichlet character associated with $K$, and such that every prime in $S$ splits in $\mathbf{Q}(\sqrt{D})$.

The significance of this is that if $f$ is a modular form of weight two associated with an elliptic curve $E$ defined over $\mathbf{Q}$ such that $L(1, f) \neq 0$, then according to the theorem of Gross and Zagier [8], the existence of such a twist implies the nonvanishing of the associated Heegner point in the twisted curve whose $L$-function is $L\left(s, E, \chi_{D}\right)$. By recent work of Kolyvagin [11], this in turn implies the finiteness of $E(\mathbf{Q})$, and of the Tate-Shafarevich group of $E$ over $\mathbf{Q}$.

The method of proof is the same as that of Theorem 2 in our paper [2]. This may be regarded as an extension to the non-CM case of the technique introduced by Goldfeld, Hoffstein and Patterson [7]. In their work, a certain Dirichlet series in an auxiliary variable $u$ is associated with an elliptic curve $E$ having complex multiplication in an imaginary quadratic field $K$. This Dirichlet series has the property that the coefficient of $D^{-u}$, where $D$ is a fundamental discriminant, is (essentially) $L\left(s, E, \chi_{D}\right)$. By differentiating with respect to $s$, and studying the pole of the resulting Dirichlet series (as a function of $u$ ), their construction would yield results similar to the above Theorem in the CM case. They obtain their Dirichlet series by applying a Galois-twisted Rankin-Selberg integral to an Eisenstein series formed with the Grössencharakter associated with $E$ on the double cover of $G L(2)$ over $K$.

In the non-CM case, the latter Eisenstein series is not available. Instead we make use of the alternative construction introduced in [2]. Let $\tilde{G}$ be the double cover of $G=G \mathrm{Sp}(4)$-the metaplectic group. The standard maximal parabolic subgroup of $G$ has Levi factor a central extension of $G L(2)$, and the double cover of $G \operatorname{Sp}(4)$ splits over this copy of $G L(2)$. This makes it possible to associate with an automorphic form $f$ on $G L(2)$ an Eisenstein series on $\tilde{G}$. We prove that this Eisenstein series has a Whittaker model, and that the Whittaker coefficients are essentially the values of $L\left(s, f, \chi_{D}\right)$.

We apply to this Eisenstein series an integral transform of a type introduced by Novodvorsky [12]. This yields a Dirichlet series in a variable $u$ in which the coefficient of $D^{-u}$, where $D$ is a fundamental discriminant, is (essentially) $L\left(s, f, \chi_{D}\right)$. Thus if we take $f$ to be the modular form of weight two associated with a modular elliptic curve, we obtain an extension of the Dirichlet series introduced in [7] to the non-CM case. It is 
possible to sieve this Dirichlet series so that only coefficients belonging to a particular congruence class and sign occur. For our purposes, it is sufficient to retain those $D<0$ which are congruent to squares modulo $4 N$. By differentiating this Dirichlet series with respect to $s$ at $s=k / 2$ and showing that there is a pole at $u=1$, we obtain the Theorem.

An improvement in technique over [2] comes from a suggestion of D. Zagier, who proposed to us that the theory of Jacobi forms [4], which are automorphic forms on the Jacobi group-the semidirect product of $G \mathrm{Sp}(4)$ by the Heisenberg group-would be a convenient context for this work. Thus the Eisenstein series on $\tilde{G}$ which we consider occurs as the coefficient of a theta function in the Fourier-Jacobi expansion of an Eisenstein series on the Jacobi group. Computing the Whittaker coefficients of the Eisenstein series on $\tilde{G}$ is reduced to computing integrals of a certain subset of the Siegel-Fourier coefficients of the Jacobi-Eisenstein series. As it turns out the use of Jacobi forms also simplifies the sieving process.

Our method will almost certainly yield the following estimates, which are however not yet proved. If the weight of $k$ is two, as in the elliptic curve case, then

$$
\sum_{\substack{0<-D<x \\ D \equiv 1 \bmod 4 m}} L^{\prime}\left(1, f, \chi_{D}\right)=c_{1} x \log x+c_{2} x+O\left(x^{3 / 5+\varepsilon}\right),
$$

where $c_{1}$ and $c_{2}$ are constants (depending on $f$ ), $c_{1}$ is nonzero, and the summation is over fundamental discriminants.

Recently M. R. Murty and K. V. Murty have informed us that they can prove a result similar to our Theorem by a different technique.

Details of this work will appear elsewhere. We would like to express our thanks to D. Zagier for his suggestion, and to D. Goldfeld for originally calling our attention to the connection between our work and that of Kolyvagin, and for helpful advice along the way.

\section{REFERENCES}

1. D. Blasius, A. Clozel and D. Ramakrishnan, Algébricité de l'action des opérateurs de Hecke sur certaines formes de Maass, C. R. Acad. Sci. Paris 305 (1987), 705-708.

2. D. Bump, S. Friedberg and J. Hoffstein, Eisenstein series on the metaplectic group and nonvanishing theorems for automorphic L-functions and their derivatives, Ann. of Math. (2) (to appear).

3. J. Coates and A. Wiles, On the conjecture of Birch and Swinnerton-Dyer, Invent. Math. 39 (1977), 223-251.

4. M. Eichler and D. Zagier, The theory of Jacobi forms, Birkhäuser, 1985.

5. D. Goldfeld, Gauss' class number problem for imaginary quadratic fields, Bull. Amer. Math. Soc. (N.S.) 13 (1985), 23-37.

6. D. Goldfeld and J. Hoffstein, Eisenstein series of 1/2-integral weight and the mean value of real Dirichlet L-series, Invent. Math. 80 (1985), 185-208.

7. D. Goldfeld, J. Hoffstein and S. Patterson, On automorphic functions of half-integral weight with applications to elliptic curves, Number Theory Related to Fermat's Last Theorem (N. Koblitz, ed.), 1982, pp,. 153-194.

8. D. Gross and D. Zagier, Heegner points and derivatives of L-series, Invent. Math. 84 (1986), 225-320. 
9. R. Howe, $\theta$-series and invariant theory, Automorphic Forms, Representations and $L$ functions, Proc. Sympos. Pure Math., vol. 33, Amer. Math. Soc., Providence, R.I., 1979, pp. $275-285$.

10. H. Jacquet, J. Shalika and I. Piatetski-Shapiro, in preparation.

11. V. Kolyvagin, On groups of Mordell-Weil and Shafarevich-Tate and Weil elliptic curves, Preprint, (1988) (Russian).

12. M. Novodvorsky, Automorphic L-functions for the symplectic group $G \mathrm{Sp}_{4}$, "Automorphic Forms, Representations and $L$-functions, Proc. Sympos. Pure Math., vol. 33, Amer. Math. Soc., Providence, R.I., 1979, pp. 87-95.

13. D. Rohrlich, Nonvanishing of L-functions for $G L(2)$, Invent. Math. (to appear).

14. G. Shimura, On the periods of modular forms, Math. Ann. 229 (1977), 211-221.

15. J. Tunnell, $A$ classical Diophantine problem and modular forms of weight $3 / 2$, Invent. Math. 72 (1983), 323-334.

16. J. Waldspurger, Correspondence de Shimura, J. Math. Pures Appl. 59 (1980), 1-132.

17. __ Sur les coefficients de Fourier des formes modulaires de poids demi-entier, J. Math. Pures Appl. 60 (1981), 375-484.

Department of Mathematics, Stanford University, Stanford, California 94305

Department of Mathematics, University of California at Santa Cruz, Santa Cruz, CALIFornia 95064

Department of Mathematics, University of Rochester, Rochester, New York 14627 
